\title{
Probing magnetar physics through high time-resolved spectroscopy of X-ray flashes
}

\author{
Israel Gian Luca* \\ INAF - Astronomical Observatory of Rome, Monte Porzio Catone (RM), I-00040, Italy \\ E-mail: gianluca@mporzio.astro.it
}

In January 1979, for the first time, recurrent short $(<100 \mathrm{~ms})$ and intense $\gamma$-ray bursts were detected from a source in the direction of the Galactic center, later named SGR1806-20. Since then, many advances have been achieved in the study of this class of high energy emitting isolated neutron stars, thought to shine in the X-rays due to the decay of the strongest magnetic fields present in the Universe ( $>10^{15}$ Gauss). In the following a number of recent results will be reported and discussed with particular emphasis on the short and intense events displayed by this class of objects. Time resolved spectroscopic studies of the flare emission from magnetars was in fact used to infer information related to the neutron star and magnetosphere structure, the emitting processes, and the confined fireball properties. In particular, within the magnetar model, the majority of these findings can be accounted for in terms of thermalised emission from the E-mode and O-mode photospheres.

High Time Resolution Astrophysics IV - The Era of Extremely Large Telescopes - HTRA-IV,

May 5-7, 2010

Agios Nikolaos, Crete, Greece

${ }^{*}$ Speaker. 


\section{MAGnetic NEutron sTARS}

Soft Gamma-ray Repeaters (SGRs; seven confirmed members) and Anomalous X-ray Pulsars (AXPs; ten confirmed members) are two classes of X-ray pulsating sources with no evidence for companion stars and first identified due to their rather different phenomenology with respect to other classes of high energy sources. The SGRs were discovered as sporadically bursting objects, while the AXPs were regarded as peculiar pulsars with long spin periods. Besides that, more recent observations unveiled several similarities between these two classes. These include rotation periods of several seconds $(\mathrm{P} \sim 2-12 \mathrm{~s})$, rapid spin down $\left(\dot{\mathrm{P}} \sim 10^{-11} \mathrm{~s} \mathrm{~s}^{-1}\right)$, large and variable X-ray luminosities, and the emission of flares and short bursts (see [1] for a review). Their spectra in the $0.1-10 \mathrm{keV}$ band are relatively soft and can be empirically fitted with a two-component model, an absorbed blackbody $(k T \sim 0.2-0.6 \mathrm{keV})$ plus a power-law $(\Gamma \sim 2-4)$. INTEGRAL observations revealed the presence of sizeable emission up to $\sim 200 \mathrm{keV}$, which accounts for up to $50 \%$ of the total flux 洞一母. Hard X-ray spectra are well represented by a power-law, which dominates above $\sim 20 \mathrm{keV}$ in AXPs.

SGRs and AXPs are currently interpreted as observational manifestations of magnetars, namely neutron stars endowed with ultra-strong magnetic fields [5, 6]. In this framework, the high energy emission of these objects, including the peculiar bursting activity, is ultimately powered by the decay of the enormous magnetic field. In order to explain the observed phenomenology, magnetars magnetic fields are expected to be $\sim 10^{14}-10^{15}$ Gauss $>B_{Q E D} \simeq 4.4 \times 10^{13}$ Gauss on the surface and one (or more) order of magnitude higher in the interior [7]-10]. This picture is compatible with the magnetic fields of $\sim 10^{14}$ Gauss inferred for SGRs and AXPs from their period and period derivative (e.g. [11]).

In the magnetar model, the magnetic field is the dominant source of free energy (orders of magnitudes higher than the rotational energy of the star), powering the persistent emission through low-level seismic activity and heating of the stellar interior [9]. When magnetic stresses build up sufficiently to crack a patch of the neutron star crust, the resulting "crustquake" ejects hot plasma particles into the magnetosphere, which results in an SGR burst [8]. Alternatively, the short SGR bursts may arise from magnetic reconnection events in the stellar magnetosphere [12]. Giant flares likely result from a sudden reconfiguration of the star's magnetic field that produces large fractures in the crust and propagates outwards through Alfvén waves of enormous power [13]. The ringing tail that follows results most likely from the part of the fireball that remains trapped in the starâĂŹs magnetosphere. The energy release in the ringing tail yields a limit for the external field of magnetars ( $>10^{14}$ Gauss) in agreement with the values inferred from spin-down dipole losses [8, 14]. The super-Eddington fluxes observed in the tails of these events are thought to be possible because of the suppression of the electron scattering cross sections in the presence of very strong magnetic fields [ [5]. In the following I will focus on a number of recent results and on the information that have been obtained by means of the study of the timing and spectral properties of the flaring activity of magnetars.

\section{Intermediate Flares}

Burst activity in SGRs occurs sporadically and is quite diverse in behavior. Burst active phases 
of SGRs vary both in total energy released and duration, tending to be concentrated into relatively narrow intervals (weeks or months) separated by relatively long periods (years) of quiescence. Since its discovery in 1979, when bursts where detected three times in three days, SGR 1900+14 remained in quiescence until 1992, when a handful of bursts were again detected within a few days [15, 16]. In 1998 the source entered an unprecedented level of activity during which more than 1000 burst were recorded within 9 months, and culminated with the $\sim 400 \mathrm{~s}$ long and rather intense 1998 August 27 giant flare event $\left(\sim 4 \times 10^{44} \mathrm{erg} \mathrm{s}^{-1}\right)$. Burst activity was detected until 1999 . Finally, after almost two years of inactivity, on 2001 April 18, an intense and long ( $\sim 40$ s) burst was detected from SGR 1900+14 [17]. The energy released by this flare (few $\times 10^{42} \mathrm{ergs}$ ) was less than that of the giant flare, though larger than that of the most common short SGR bursts. Consequently the flare was dubbed an "intermediate flare" [18]. Indeed, looking back at the burst history of SGR 1900+14 and other SGRs, it was realized that a handful of similar events, characterized by a longer duration (few seconds-few tens of seconds) and a higher fluence than those characterizing short bursts, were present. These bursts were then grouped into the class of intermediate flares likely forming a continuum in terms of duration and fluences [19].

A number of broad-band spectroscopic studies of short and intermediate flares were carried out in the past by different missions. The study of the cumulative spectral properties of 100 bursts from SGR 1806-20 in the 5-200 keV energy range showed that the number of photons in the burst spectra was remarkably stable in spite of the large intensity spread (a factor of 50) and that the low-energy (below $15 \mathrm{keV}$ ) data were inconsistent with the an OTTB model that provided a good fit to the high-energy portion of the spectrum [20, 21]. Qualitatively similar spectral properties were measured during a bright intermediate flare from SGR 1900+14 using HETE-2 data, which also showed that the OTTB model largely overestimated the flux at low energies $(<15 \mathrm{keV}$, [19]). The broadband spectrum (7-100 keV) was best fitted by the sum of two blackbody (BB) laws.

Feroci et al. (2004,[2]) analyzed the 1.5-100 keV BeppoSAX spectral properties of 10 short burst from SGR 1900+14, further confirming that the best fit was obtained by means of an alternative spectral model such as two blackbodies or a cut-off power law.

\subsection{The March 2006 Burst Storm from SGR 1900+14}

In 2006 March the Soft Gamma-ray Repeater SGR 1900+14 resumed its bursting activity after about 2 years of quiescence. The Swift mission observed a the intense burst "storm" which lasted for $\sim 30 \mathrm{~s}$, when Swift was pointing at the source with the narrow field of view instruments. More than 40 bursts were detected both by BAT and by XRT, seven of which are rare intermediate flares (IFs): several times $10^{42}$ ergs were released (see [23] for details).

With respect to the former studies of the broad-band spectrum of similar events, the Swift observation of 2006 March 29 has the advantage of a large number of events ( $>40$ between short bursts and IFs), therefore largely improving the statistics over which the detailed analysis can be carried out. The impact of the increased statistics and time resolution is evident when comparing the Swift results with previous ones. On 2001 July 2, the HETE-2 instruments recorded a $3.5 \mathrm{~s} \mathrm{IF}$ from SGR $1900+14$ and a time resolved $(\Delta t>30 \mathrm{~ms})$ spectral analysis was carried out in the 2$100 \mathrm{keV}$ range [19]. They found that the $2 \mathrm{BB}$ model fitted the spectra well, the higher temperature blackbody $\left(\mathrm{BB}_{\text {hard }}\right)$ evolving in a manner consistent with a shrinking emitting region, the lower temperature one $\left(\mathrm{BB}_{\mathrm{soft}}\right)$ showing a constant radius. Figure $\mathrm{\theta}$ includes both the results obtained 


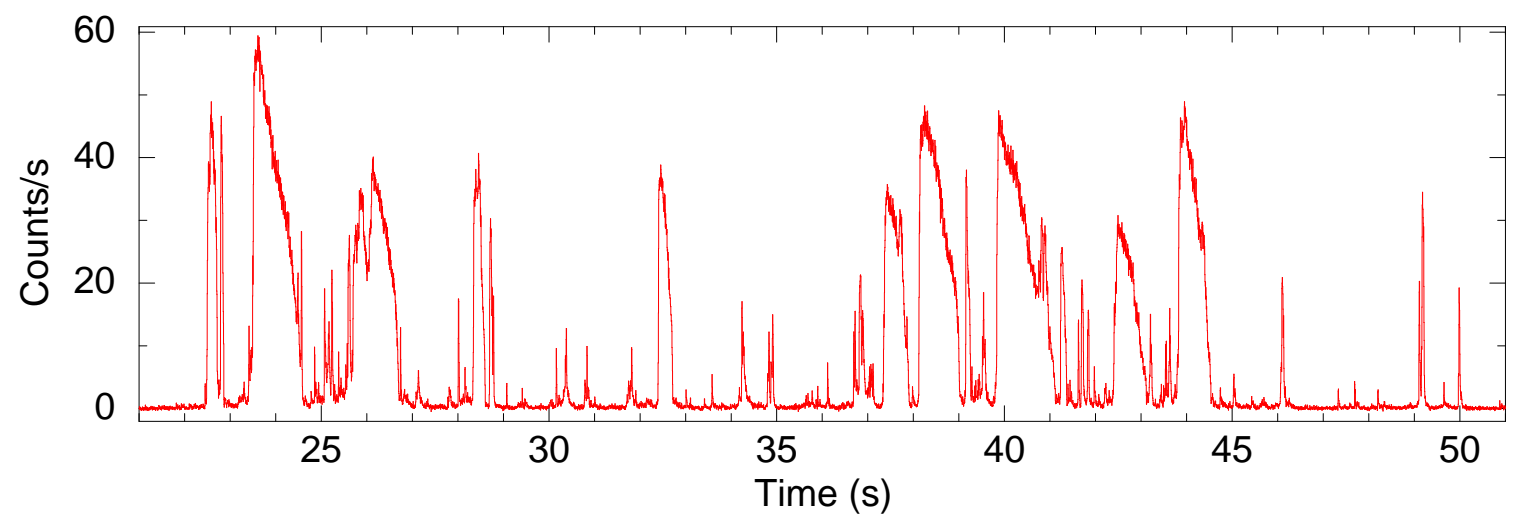

Figure 1: Swift BAT light curves obtained during the burst "storm” of 2006 March 29.

from the Swift and HETE-2 data: clearly, the Swift data fill in those regions on the $k T-R^{2}$ plane, which remained unexplored with HETE-2.

A number of properties can be inferred: a) the Swift data populate, almost homogeneously, all temperatures between $\sim 2$ and $12 \mathrm{keV}$, and the HETE-2 measurements (black diamonds in Figure 2) can be regarded as a subset of them; b) the distribution is such that a sharp edge between the populated regions and the rest of the $k T-R^{2}$ plane is clearly present, with a cut-off (or sharp turn) in the distribution of $R_{\mathrm{BBh}}$ at $k T_{\mathrm{h}} \approx 12-13 \mathrm{keV}$. This sharp edge provides an estimate of the typical size of the relevant emitting regions (by assuming a reference distance of $10 \mathrm{kpc}$; [24, 25]): 30-200 km range for the $\mathrm{BB}_{\mathrm{s}}$ component and $3-30 \mathrm{~km}$ range for the $\mathrm{BB}_{h}$ one. c) there is an additional turn in the $\mathrm{BB}_{\mathrm{h}}$ component size between 6 and $16 \mathrm{~km}$, at approximately $10 \mathrm{keV}$ (see Figure 2), and d) there is a strong correlation between $k T$ and $R_{B B}^{2}$ in the brightest phases of the IFs, corresponding to $\mathrm{BB}$ temperatures $\leq 10 \mathrm{keV}$. This correlation is consistent with a power-law with index of about -3 (while, were the luminosity the same for all events, the relationship expected for a BB component would be $\mathrm{y}=\mathrm{x}^{-4}$, where $x=k T$ and $y=R_{B B}^{2}$ ).

The sharp edge in the distribution of the $2 \mathrm{BB}$ parameters suggests that a saturation effect occurs. As we can see from right panel of Figure 目, as long as both BB component are below $\sim 3 \times 10^{40} \mathrm{erg} \mathrm{s}^{-1}$, their two luminosities are strongly correlated ( $L\left(B B_{\text {hard }}\right)$ versus $L\left(B B_{\text {soft }}\right)$ is well fitted by a power law with index $0.7 \pm 0.3$ at $1 \sigma$ c.l.). This correlation, which suggests a physical link between the two BB components, was already noticed by Feroci and collaborators from BeppoSAX data (filled squares in Figure 2, right panel; [22]). It is apparent that these data are fully consistent with those obtained with Swift and can be regarded as a subsample of the latter. The correlation no longer holds for luminosity values above $\sim 3 \times 10^{40} \mathrm{erg} \mathrm{s}^{-1}$. Above this value, the luminosity of the $\mathrm{BB}_{\text {soft }}$ component reaches a maximum in the (7-14) $\times 10^{40} \mathrm{erg} \mathrm{s}^{-1}$ range, while that of the $\mathrm{BB}_{\text {hard }}$ continues to increase up to $\sim 3 \times 10^{41} \mathrm{erg} \mathrm{s}^{-1}$. Clearly, this previously unknown behavior strengthens the idea of a saturation mechanism in the burst emission.

In the following, we will concentrate on the information (mainly concerning the brightest part of IFs) which can be inferred from spectral analysis of IFs.

i) the existence of two blackbody components, the harder one $\left(k T_{\mathrm{h}} \sim 7-11 \mathrm{keV}\right)$ having a systematically smaller radius $\left(R_{\mathrm{BBh}} \leq 25 \mathrm{~km}\right)$ than the softer one $\left(R_{\mathrm{BBs}} \sim 25-100 \mathrm{~km}\right)$. 

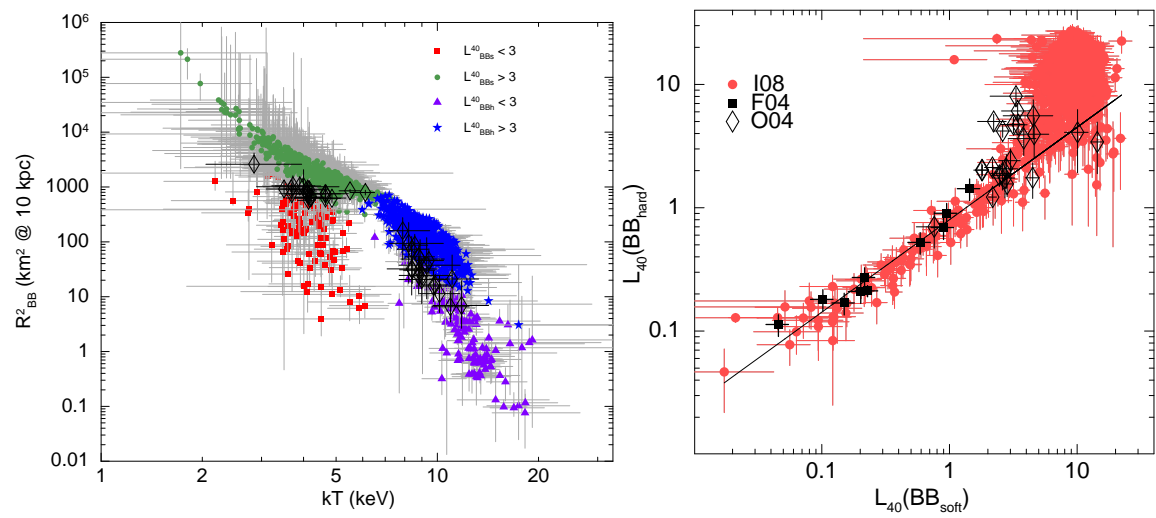

Figure 2: Swift BAT time-resolved spectral results obtained during the burst "storm" of 2006 March 29. Left panel: square of radii of the two blackbodies as a function of their temperatures for the whole $\sim 30 \mathrm{~s}$ BAT dataset. Red filled squares and dark green filled circles mark the $\mathrm{BB}_{\text {soft }}$ component for luminosities below and above $3 \times 10^{40} \mathrm{erg} \mathrm{s}^{-1}$, respectively. Violet filled triangles and blue filled stars are the same quantities as above for the $\mathrm{BB}_{\text {hard }}$ component. Black diamonds marks the HETE measurements obtained for the $2001 \mathrm{IF}$ ([19]=004). We also plot two representative power-laws for $R^{2}=k T^{\alpha}$, with $\alpha$ equal -3 and -4 (the latter corresponding to the expected relation for a pure BB). Right panel: Time-resolved bolometric luminosity of the $\mathrm{BB}_{\text {soft }}$ versus that of the $\mathrm{BB}_{\text {hard }}$ (in units of $10^{40}$ ). The solid line marks the power-law relation $L_{\text {soft }}^{40}=\left(L_{\text {hard }}^{40}\right)^{\alpha}$ with $\alpha=0.70 \pm 0.03$ (1 $\sigma$ c.l.) obtained by fitting points in the $0.01-3 L_{\text {soft }}^{40}$ range. Filled squares represent the measurements for short bursts ([22] $=\mathrm{F} 04$; adapted from [23] $]=\mathrm{I} 08)$.

This first finding shows that different thermal components originate from different regions around the NS, ranging from its surface (at $R<R_{\mathrm{NS}}$ ) to well up in the magnetosphere, at a height of several stellar radii. The maximum observed flare luminosity is $\sim 3 \times 10^{41} \mathrm{erg} \mathrm{s}^{-1}$, attained by the hard blackbody component at an effective temperature of $\sim 10 \mathrm{keV}$ and radius of $\sim 15 \mathrm{~km}$. This matches well the magnetic Eddington luminosity, $L_{\mathrm{Edd}, \mathrm{B}}$, at that same radius, for a surface dipole field $\simeq 8 \times 10^{14} \mathrm{G}$ (this value of the B-field is very close to that deduced from the spindown rate of SGR 1900+14), where (cfr. [5], 8]):

$$
L_{\mathrm{Edd}, \mathrm{B}}(r) \simeq 2 L_{\mathrm{Edd}}\left(\frac{B}{10^{12} \mathrm{G}}\right)^{4 / 3} \approx 2 \times 10^{40}\left[\frac{B}{B_{\mathrm{QED}}}\right]^{4 / 3}\left(\frac{R}{R_{\mathrm{NS}}}\right)^{2 / 3} \mathrm{erg} \mathrm{s}^{-1} .
$$

If radiation originated from a trapped hot fireball this would be in line with the theoretical expectation that the radiative efficiency of a magnetic confined fireball never exceeds (to within a small factor) the magnetic Eddington flux [8, 14]. The radius and temperature that we inferred at maximum burst luminosity are also in good agreement with the prediction for the emission coming from a trapped fireball ([8])

Most remarkably, the luminosity of the soft component can be larger than $10^{40} \mathrm{erg} \mathrm{s}^{-1}$ out to $\mathrm{R} \sim 100 \mathrm{~km}$. For $\mathrm{R}>(30-40) \mathrm{km}$ this value exceeds the magnetic Eddington luminosity at the corresponding radius (for a dipole field geometry). This suggests that magnetospheric confinement plays an active role at those distances, with magnetic stresses balancing radiation forces.

ii) the existence of a clear correlation between the luminosities of the two components up to $\sim 3 \times 10^{40} \mathrm{erg} \mathrm{s}^{-1}$, above which value the luminosity of softer blackbody shows signs of saturation, while that of the harder blackbody still grows up to a few times $10^{41} \mathrm{erg} \mathrm{s}^{-1}$. 
This second finding implies that, in the luminosity range $10^{40}-10^{41} \mathrm{erg} \mathrm{s}^{-1}$, the total radiation energy is divided almost equally between the two components. Combining the information of the two panels of Figures 2 we can see that, for a given luminosity $\leq 10^{41} \mathrm{erg} \mathrm{s}^{-1}$, there are two separate emission regions: a smaller and hotter one, whose radiating area suggests emission from (part of) the NS surface with a relatively high effective temperature $(10-12 \mathrm{keV})$, plus a second, possibly magnetospheric region, with significantly larger emitting area and lower effective temperature (3-7 keV). The hotter component attains the highest luminosities, i.e. $>10^{41} \mathrm{erg} \mathrm{s}^{-1}$, as its radius grows slightly and the effective temperature decreases. On the other hand, the colder component is characterized by a minimum blackbody radius of $\sim 25 \mathrm{~km}$ and maximum temperature of $\sim 7 \mathrm{keV}$.

A possible interpretation involves the different way in which photons with ordinary $(\mathrm{O})$ or extraordinary (E) polarization mode (a property introduced by the presence of a birefringent medium, such as the magnetic field; [26]) propagate across the magnetosphere. Since the scattering cross section of E-mode photons is much reduced in presence of strong magnetic fields, E-mode photons have a scattering photosphere which is located much closer to the NS than that of O-mode photons. On the other hand, in supercritical magnetic field $\left(B>B_{\mathrm{QED}}\right)$, E-mode photons have a non-negligible probability of splitting (and switching to the O-mode). The probability is a strong function of energy, so that E-mode photons of high energy cannot travel far from their scattering photosphere before splitting into O-mode photons. As a consequence of Compton scattering (and, with lower efficiency, photon merging), a fraction of O-mode photons can also switch back to the E-mode. Therefore, the two modes are effectively coupled and are advected at comparable rates as long as mode switching is efficient: an approximately equal distribution of energy in the two modes is thus to be expected [8, 12]. This may suggest that the two observed spectral components reflect the population of photons in the two polarization modes and, thus, the regions from which they are emitted.

The sharp and narrow gap between the emitting regions of the two BBs, at a radius of $(\sim 20$ $30 \mathrm{~km}$ ), can be interpreted as the signature of the presence of an E-mode splitting photosphere; this represents the surface (at radius $R=R_{\mathrm{QED}}$ ) below which the magnetic field is supercritical ( $B \geq B_{\mathrm{QED}}$ ), photon splitting is efficient and the two polarization modes can remain coupled. Emode photons can stream freely to the observer from the splitting photosphere (at radius $R_{\mathrm{QED}} \sim 20$ $30 \mathrm{~km}$ ), which then would naturally define a maximum size for the emission region of radiation in this polarization mode. On the other hand, O-mode photons could not originate from regions internal to the splitting photosphere, as their scattering optical depth is still much larger than unity within that volume.

iii) the existence of a correlation between temperature and blackbody radius, which holds for the most luminous parts of the flares (approximately for $L_{\text {tot }}>4 \times 10^{40} \mathrm{erg} \mathrm{s}^{-1}$ ).

Finally, our third finding is the apparent correlation between the surface and temperature of the spectral components at different luminosities. This behavior does not have a clear interpretation. As shown in Figure 2, the top part of the data strip is well fit by a power-law $\Sigma \propto T_{\mathrm{BB}}^{-3}$, where $\Sigma$ is the radiating blackbody surface. Since $\Sigma T_{\mathrm{BB}}^{4}=L$, this implies $T_{\mathrm{BB}} \propto L$. Therefore, along lines of constant luminosity, the radiating surface would scale like $T^{-4}$, i.e. steeper than the data points (on the edge of the allowed region in the $\mathrm{kT}$ versus $\mathrm{R}^{2}$ plane). The $\Sigma T^{3}=$ const relation that implies a 

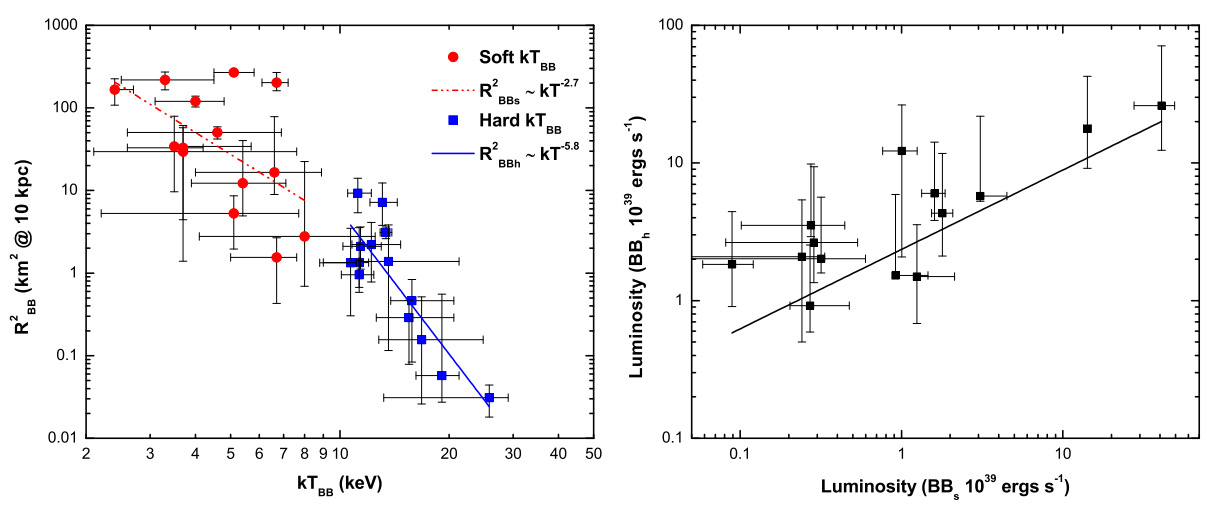

Figure 3: Left panel: square of the radii of the $\mathrm{BB}+\mathrm{BB}$ model components as a function of their temperatures. The red circles and blue squares denote the soft and hard $k T$ values, respectively. The soft $k T$ values show an anti-correlation of $\rho=-0.5, P=0.06$ with $R_{B B_{s}}^{2}$, and the hard $k T$ shows a strong anti-correlation of $\rho=-0.7, P=0.01$ with $R_{B B_{h}}^{2}$. Right panel: bolometric luminosity of the hard BB component $\left(L_{\text {hard }}\right)$ versus that of the soft BB component $\left(L_{\mathrm{soft}}\right)$ in units of $10^{39} \mathrm{ergs} \mathrm{s}^{-1}$. The solid line is the powerlaw fit which gives the relation $L_{\mathrm{soft}}=\left(L_{\mathrm{hard}}\right)^{\alpha}$ where $\alpha=0.6 \pm 0.1$ (from [27]).

roughly constant number of emitted photons per unit time.

At the highest luminosities ( $\geq 10^{41} \mathrm{erg} \mathrm{s}^{-1}$ ) of the flares, where the softer blackbody saturates, the observed correlation involves both components. It is tempting to attribute this to the fact that, in this luminosity range, Comptonization, and/or adiabatic losses play a dominant role in the formation of the emerging spectrum, both processes being characterized by conservation of the photon number (photon splitting should have only a minor impact). This is in agreement with the prediction by [8], that at effective temperatures less than $\sim 10 \mathrm{keV}$ photon splitting would not be efficient in maintaining a pure Planck spectrum for both modes.

\subsection{Further Observations: the case of SGR 0501+4516 and 1E 1547.0-5408}

The results outlined in the previous section are certainly interesting, though they should be confirmed and tested through the study of similar events (IFs or bursts) with sufficient statistics detected both from the same source or from other magnetars.

During 2008 three outbursts from three different magnetars were detected, namely SGR 162741, SGR 0501+4516 and 1E 1547.0-5408. In all cases the outburst onsets were studied by Swift after the detection of one or more bursts from the sources in the BAT (for details see [28 - 30]). In all cases the statistics collected by BAT was not sufficient (both in terms of detected bursts and statistics within each burst) to allow a detailed time-resolved study of the spectral parameters during the bursts. However, in the case of SGR 0501+4516 more than 50 bursts were identified out of which 18 had enough counts to carry out a relatively detailed time-averaged spectral analysis ([27]; see Figure 3). Among the several different spectral models used, the cut-off powerlaw and the twoblackbody models provided the best fit in the $15-150 \mathrm{keV}$ energy range. In particular, the mean hard and soft blackbody temperatures are found to be $k T=12.8 \pm 0.7 \mathrm{keV}$ and $k T=4.6 \pm 0.5 \mathrm{keV}$ for the $\mathrm{BB}_{\text {soft }}$ and $\mathrm{BB}_{\text {hard }}$ components, respectively. Moreover, they are anti-correlated with the square of 

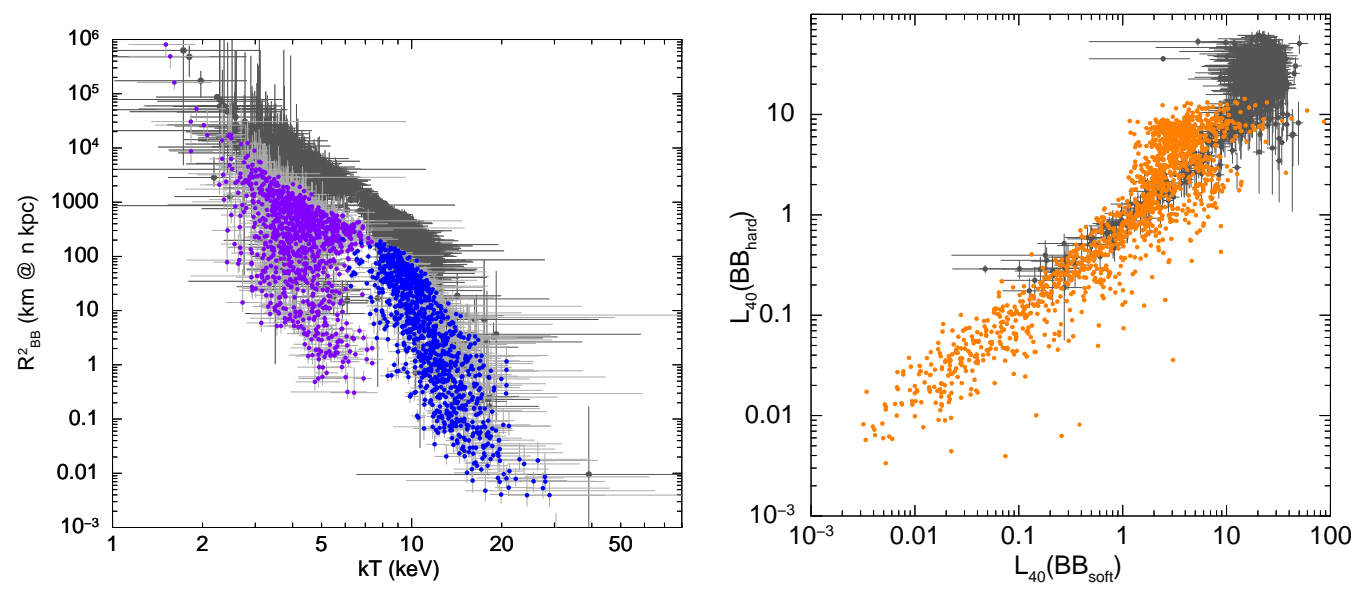

Figure 4: Similar to Figure 2 but for SGR 1900+14 and 1E 1547.0-5408 together. Radii and luminosities (the latter without uncertainties in order to better appreciate the comparison with the corresponding quantities inferred for SGR 1900+14) are normalized to the distances of $15 \mathrm{kpc}$ for SGR $1900+14$ and $4.5 \mathrm{kpc}$ for 1E 1547.0-5408 (adapted from [32, 33]). Left panel: grey points refer to SGR 1900+14, while those in blue and violet mark the hard and soft blackbody components of 1E 1547.0-5408, respectively. Right panel: grey points refer to SGR $1900+14$.

the radii of the hard and soft emitting regions $\left(R_{B B_{h}}\right.$ and $\left.R_{B B_{s}}\right)$ as $R_{B B_{h}}^{2} \propto k T^{-5.8}$ and $R_{B B_{s}}^{2} \propto k T^{-2.7}$, respectively, similarly to the case of SGR $1900+14([27])$. However, by comparing datapoints in Figure 2 and 3, it is evident that the "saturation" effect was not reached during SGR 0501+4516 bursts, or at least not observable within the time-averaged spectra.

It was only in January 2009 that a large outburst was detected from 1E 1547.0-5408 after only three months from the previous one (see [32, 33] for more details). 1E 1547.0-5408 is very peculiar magnetar, first discovered in 1980 with the Einstein X-ray satellite [34], but studied in detail for the first time only in 2007 [35]. Using data collected in June 2007 with both the Parkes radio telescope and the Australia Telescope Compact Array, the source was unambiguously classified as a transient magnetar by measuring $P \sim 2.069 \mathrm{~s}$ and $\dot{P} \sim 2.3 \times 10^{-11} \mathrm{~s} / \mathrm{s}$ [36]. During January 22,2009 , the source entered in a rather strong state of burst activity (first detected by Swift BAT), accompanied by a strong enhancement of the continuum $\mathrm{X}$-ray flux, which culminated when more than 200 bursts (some of which were classified as IFs) were recorded by the INTEGRAL SPI instrument in a few hours [37]. Moreover, the distance to 1E 1547.0-5408 was independently found to be in the $4-5 \mathrm{kpc}$ range on the basis of the possible association with the supernova remnant G327.24-0.13 [35], and, more convincingly, from the analysis of multiple expanding rings around the source [38].

Several tens of bursts were also detected by Swift BAT for several weeks since the outburst onset, ensuring a reliable dataset to carry out time-resolved spectroscopy of the brightest events and to prove (or disprove) the findings inferred for SGR 1900+14. About 1300 spectra were analysed, similarly to the case of SGR 1900+14, in an automatic and systematic fashion (see [32] for the details). The radius and luminosity values were corrected for the distance of the source (we assumed $4.5 \mathrm{kpc}$ ) and were compared with those of SGR 1900+14 already presented in Figure 2 (rescaled to $15 \mathrm{kpc}$ with respect to Figure 2). Figure 7 summarizes the comparison between the two sources. A number of properties can be immediately noticed: i) the datapoint distributions for the two sources 
in the $k T$ versus $R_{B B}^{2}$ plane are very similar, ii) the two distributions have a significant relative shift both in $k T$ and $R_{B B}^{2}$, which could partially be caused by the different distances of the two sources iii) the "saturation" effect (the sharp edge in the distribution of datapoints described by the $R^{2}=k T^{-3}$ relation) and the cut-off at around (10-15) $\mathrm{keV}$ are present in both sources, iv) the "saturation" is also clearly present in the $L_{\text {hard }}$ versus $L_{\text {soft }}$ plane, where the $L_{\text {soft }}$ inferred for 1E 1547.0-5408 saturates at a flux of about $2 \times 10^{40} \mathrm{erg} / \mathrm{s}$, a factor of 10 smaller then that of SGR $1900+14$ [32].

While the greatest part of the above listed properties are not that unexpected, it is worth commenting on the overall shift of the $L_{\text {soft }}$ distributions. In fact, according to Eq.2.1 the saturation occurs when the magnetic field ability to confine the fireball is itself saturated. It is therefore strictly dependent on the value of the magnetic field at the given radius. If this hypothesis is correct, then it would be possible to infer a number of properties comparing the distributions and checking that the so obtained results quantities (such as $B$ or $d$ ) are in agreement with those obtained by means of different approaches (e.g. from timing analysis or other techniques). In particular, Eq.2.1 can be rewritten in the following form

$$
\left(\frac{d}{\mathrm{kpc}}\right) \simeq 0.4 \times\left(\frac{F_{\max }}{10^{-5} \mathrm{erg} / \mathrm{cm}^{2} / \mathrm{s}}\right)^{-1 / 2}\left(\frac{k T}{\mathrm{keV}}\right)^{5 / 4}\left[\frac{B_{\text {surf }}}{10^{14} \mathrm{G}}\right]^{1 / 4}\left(\frac{R_{\mathrm{ss}}}{10 \mathrm{~km}}\right)^{5 / 8}
$$

where the flux and the distance have been explicitly reported (decoupling them from $L$ and $R$ ). The importance of this relationship is that, if the magnetic field strength has been inferred by means of different techniques (timing analysis as an example) it is then possible to derive an approximated value for the distance by using the saturation flux value recorded for the sources. Viceversa, if the distance is known from other considerations it is then possible to infer the magnetic field strength. By inserting the (unabsorbed) bolometric fluxes (3 and 6 times $10^{-5} \mathrm{ergs} \mathrm{cm}^{-2} \mathrm{~s}^{-1}$ for SGR 1900+14 and 1E 1547.0-5408, respectively) and the $k T$ values corresponding to the cut-off regions ( $\sim 13 \mathrm{keV}$ and $\sim 11 \mathrm{keV}$ for SGR 1900+14 and 1E 1547.0-5408, respectively) in relation (2.2) above, the following distance estimates are obtained: $d_{S G R 1900} \sim 8.5 \mathrm{kpc}$ and $d_{1 E 1547} \sim 4.0 \mathrm{kpc}$. Note that bona fide values of the magnetic field strength of $\sim 5 \times 10^{14} \mathrm{G}$ and $\sim 2.2 \times 10^{14} \mathrm{G}$ were assumed for SGR 1900+14 and 1E 1547.0-5408, respectively [39. 30].

It is worth noticing that the distance to SGR $1900+14$ is highly uncertain, with only a lower limit of $5 \mathrm{kpc}$ and a maximum possible distance in the $12-15 \mathrm{kpc}$ range [24, 25]. On the other hand, 1E 1547.0-5408 has a reliable distance measurement obtained in two independent ways, SNRassociation and dust (expanding) rings, both pointing to a distance in the $4-5 \mathrm{kpc}$ range. These numbers corroborate the idea of using the saturation observed in magnetars as a powerful and alternative way of assessing either the distance or the magnetic field strength if one of the two quantities is already inferred independently.

\section{Acknowledgments}

This research is based on observations with the NASA/UK/ASI Swift mission. Authors acknowledge the support from ASI (ASI/INAF contracts AAE DA-044, DA-006 and DA-017 ). GLI thanks Dr. H. Kumar for providing Figure 3 with high resolution. 


\section{References}

[1] S. Mereghetti, The strongest cosmic magnets: Soft Gamma-ray Repeaters and Anomalous X-ray Pulsars, A\&A Rev. 15 (225) 2008 [astro-ph/ 0804 . 0250]

[2] L. Kuiper, W. Hermsen, \& M. Mendez, Discovery of Hard Nonthermal Pulsed X-Ray Emission from the Anomalous X-Ray Pulsar IE 1841-045, ApJ 613 (1173) 2005 [astro-ph / 0404582 ]

[3] S. V. Molkov, A. M. Cherepashchuk, A. A. Lutovinov, et al., A Hard X-ray Survey of the Sagittarius Arm Tangent with the IBIS Telescope of the INTEGRAL Observatory: A Catalog of Sources, Astronomy Letters 30 (534) 2004

[4] P. R. den Hartog, W. Hermsen, L. Kuiper, et al., NTEGRAL survey of the Cassiopeia region in hard X rays, A\&A 451 (587) 2006 [astro-ph/ 060164 4]

[5] B. Paczynski, GB 790305 as a very strongly magnetized neutron star, Acta Astronomica 42 (145) 1992

[6] R. C. Duncan, \& C. Thompson, Formation of very strongly magnetized neutron stars - Implications for gamma-ray bursts, ApJL 392 (L9) 1992

[7] C. Thompson, \& R. C. Duncan, Neutron star dynamos and the origins of pulsar magnetism, ApJ $\mathbf{4 0 8}$ (194) 1993

[8] C. Thompson, \& R. C. Duncan, The soft gamma repeaters as very strongly magnetized neutron stars I. Radiative mechanism for outbursts, MNRAS, 275 (255) 1995

[9] C. Thompson, \& R. C. Duncan, The Soft Gamma Repeaters as Very Strongly Magnetized Neutron Stars. II. Quiescent Neutrino, X-Ray, and Alfven Wave Emission, ApJ 473 (322) 1996

[10] C. Thompson, M. Lyutikov, \& S. R. Kulkarni, Electrodynamics of Magnetars: Implications for the Persistent X-Ray Emission and Spin-down of the Soft Gamma Repeaters and Anomalous X-Ray Pulsars, ApJ 574 (332) 2002 [astro-ph / 0110677 ]

[11] D. R. Lorimer, \& M. Kramer, Handbook of Pulsar Astronomy. Cambridge, UK, Cambridge University Press 2004

[12] M. Lyutikov, Radio Emission from Magnetars, ApJ 580 (L65) 2002 [astro-ph/ 0206439 ]

[13] S. J. Schwartz, S. Zane, R. J. Wilson, et al., The Gamma-Ray Giant Flare from SGR 1806-20: Evidence of Crustal Cracking via Initial Timescales, ApJL 627 (L129) 2005 [astro-ph/ 0504056 ]

[14] C. Thompson, R. C. Duncan, The Giant Flare of 1998 August 27 from SGR 1900+14. II. Radiative Mechanism and Physical Constraints on the Source, ApJ 561 (980) 2001

[15] E. P. Mazets, et al., Catalog of cosmic gamma-ray bursts from the KONUS experiment data. I, Ap\&SS 80 (3) 1981

[16] C. Kouveliotou, et al., Recurrent burst activity from the soft gamma-ray repeater SGR $1900+14$, Nature 362 (728) 1993

[17] C. Guidorzi, et al., Comparative study of the two large flares from SGR1900+14 with the BeppoSAX Gamma-Ray Burst Monitor, A\&A 416 (297) 2004 [astro-ph/ 0312062 ]

[18] C. Kouveliotou, et al., Multiwavelength Observations of the Soft Gamma Repeater SGR 1900+14 during Its 2001 April Activation, ApJL 558 (L47) 2001 [astro-ph/ 0107170 ]

[19] J. F. Olive, et al., Time-resolved X-Ray Spectral Modeling of an Intermediate Burst from SGR 1900+14 Observed by HETE-2 FREGATE and WXM, Ap.J. 616 (1148) 2004 [astro-ph/0403162] 
[20] J. G. Laros, et al., The soft gamma-ray burst GB790107, Nature 322 (152) 1986

[21] Fenimore, E. E., J. G. Laros, \& A. Ulmer, The X-ray spectrum of the soft gamma repeater 1806-20, ApJ 432 (742) 1994 [astro-ph/9405031]

[22] M. Feroci, et al., Broadband X-Ray Spectra of Short Bursts from SGR 1900+14, ApJ 612 (408) 2004 [astro-ph/0405104]

[23] G. L. Israel, et al., 2008, A Swift Gaze into the 2006 March 29 Burst Forest of SGR 1900+14, ApJ 685 (1114) 2008 [astro-ph/0805.3919]

[24] F. J. Vrba, et al., The Discovery of an Embedded Cluster of High-Mass Stars near SGR 1900+14 Ap.J. 533 (L17) 2000 [astro-ph/ 0002530 ]

[25] D. L. Kaplan, et al., Constraining the Proper Motions of Two Magnetars, A.J. 137 (354) 2009 [astro-ph/0810.4184]

[26] P. Mészáros, W. Nagel, \& J. Ventura, Exact and approximate solutions for the one-dimensional transfer of polarized radiation, and applications to X-ray pulsars, ApJ 238 (1066) 1980

[27] H. S. Kumar, A. I. Ibrahim, S. Safi-Harb, Swift-BAT Observations of the Recently Discovered Magnetar SGR 0501+4516, ApJ 716 (97) 2010 [astro-ph/10 04 . 4036]

[28] P. Esposito, et al., The 2008 May burst activation of SGR1627-41, MNRAS 390 (L34) [astro-ph/0807.1658]

[29] Rea, N., et al., The first outburst of the new magnetar candidate SGR0501+4516, MNRAS 396 (2419) 2009 [astro.ph/0904.2413]

[30] G. L. Israel, et al., The 2008 October Swift detection of X-ray bursts/outburst from the transient SGR-like AXP IE 1547.0-5408, MNRAS in press 2010a, astro-ph/1006.2950

[31] A. L. Watts, et al., Photospheric radius expansion during magnetar bursts, ApJ 719 (190) 2010 [astro-ph/1006.2214]

[32] G. L. Israel, et al., in preparation 2010b

[33] L. Vetere, et al., in preparation 2010

[34] R. C. Lamb, \& T. H. Markert, X-ray emission from supernova remnants near gamma-ray sources, ApJ 244 (94) 1981

[35] J. D. Gelfand, \& B. M. Gaensler, The Compact X-Ray Source 1E 1547.0-5408 and the Radio Shell G327.24-0.13: A New Proposed Association between a Candidate Magnetar and a Candidate Supernova Remnant, ApJ 667 (1111) 2007 [astgro-ph/ 0706.1054 ]

[36] F. Camilo, et al.,1E 1547.0-5408: A Radio-emitting Magnetar with a Rotation Period of 2 Seconds, ApJ 666 (L93) 2007 [astro-ph/ 0708 .0002]

[37] S. Mereghetti, et al., Strong Bursts from the Anomalous X-Ray Pulsar 1E 1547.0-5408 Observed with the INTEGRAL/SPI Anti-Coincidence Shield, ApJ 696 (L74) 2009 [astro-ph/0903.1974]

[38] A. Tiengo, et al., The Dust-scattering X-ray Rings of the Anomalous X-ray Pulsar 1E 1547.0-5408, ApJ, 710 (227) 2010 [astro-ph/0911.3064]

[39] P. M. Woods, et al., Variable Spin-Down in the Soft Gamma Repeater SGR 1900+14 and Correlations with Burst Activity, ApJ 524 (L55) 1999 